\title{
STATISTICAL CONNECTIONS AMONG WATER VAPOR MEGAMASERS
}

\author{
A. S. WILSON \\ Astronomy Department, University of Maryland College Park, MD 20742, U.S.A. \\ and \\ Space Telescope Science Institute, 3700 San Martin Drive, Baltimore, MD 21218, \\ U.S.A.
}

J. A. BRAATZ
Center for Astrophysics, 60 Garden Street, MS 42, Cambridge, MA 02138, U.S.A.

AND

C. HENKEL

Max-Planck-Institut für Radioastronomie, Auf dem Hügel 69, D-53121 Bonn, Germany

\begin{abstract}
We review correlations between water megamaser emission and other properties of AGN. Galaxies detected as water megamaser emitters tend to be nearer, brighter at mid-infrared and possibly centimeter wavelengths, and more highly inclined than undetected galaxies. Only Seyfert $2 \mathrm{~s}$ and LINERs (no Seyfert 1s) are detected. It is argued that the primary parameter determining whether an active galaxy is detectable as an $\mathrm{H}_{2} \mathrm{O}$ megamaser is the gas column along our line of sight to the nucleus.
\end{abstract}

\section{Introduction}

Detectable water vapor megamasers are rare objects. They appear to be associated exclusively with active galaxies and, even there, the detection rate is small - about $6 \%$ in the samples described in this paper. There are two extreme types of explanation possible for this rarity. It could be that all active galactic nuclei (AGN) possess the required physical characteristics to generate maser emission, but we only see a small fraction because of anisotropy in the maser emission. The most obvious such model has the masing gas in a thin disk, with the large amplification from this disk gas causing us to see significant maser emission only when our line of sight is close to the disk plane. The VLBA images of water vapor masers in NGC 4258 support such a scenario (Miyoshi et al. 1995). Alternatively, it could be that certain special physical properties are required for significant maser emission; a fraction of active galaxies would possess these physical properties while the rest would not. Examples of relevant properties might be a nuclear hard X-rays source (to heat the dense circumnuclear molecular gas to temperatures at which water is abundant, e.g. Neufeld, Maloney \& Conger 1994) and a nuclear radio continuum source (to provide seed photons for maser amplification, e.g. Herrnstein et al. 1997).

We can address this question by searching for correlations between water maser and other properties, especially isotropic ones. An absence of correlation between maser luminosity and any isotropic property, together with evidence from VLBI mapping that the masers preferentially arise in edge-on disks, would favor the former scenario. Conversely, a strong correlation between maser luminosity and one or more isotropic properties, together with evidence that the masing gas is more randomly distributed, would support the latter. Of course, there is a whole continuum of possibilities between these two extreme models.

In this paper, we describe a study of the statistical properties of water vapor megamasers, based primarily on the recently completed Maryland-Bonn survey (Braatz et al. 1996a [Paper I], 1997 [Paper II] ). Section 2 describes the samples studied for water vapor maser emission, while Section 3 
discusses the results. Further details of the statistical study may be found in Paper II. Conclusions are summarized in Section 4.

\section{The Samples Observed}

The samples studied were selected from the catalogs of Véron-Cetty \& Véron (1991) and Huchra (1993). We observed a 'distance-limited' sample - all Seyferts and LINERs in these catalogs with recessional velocity below $7,000 \mathrm{~km} \mathrm{~s}^{-1}$ - and a 'magnitude-limited' sample - all Seyferts and LINERs in these catalogs with $\mathrm{m}_{b}<14.0$. We also observed many galaxies with $\mathrm{m}_{b}>14.0$ and used $\mathrm{m}_{b}=14.5$ as our magnitude cut-off in Paper II, even though some galaxies with $14.0<\mathrm{m}_{b}<$ 14.5 were missed. There are 278 galaxies in the 'distance-limited' sample and 241 in the 'magnitudelimited' (with $\mathrm{m}_{b}<14.5$ ) sample. Both samples together comprise 299 galaxies; about 60 other AGN (e.g. radio galaxies) were also observed. In our survey (conducted with the Effelsberg $100 \mathrm{~m}$ and Parkes 64m telescopes, see Paper I), 10 new magamasers were detected, and the total number of maser-detected galaxies in the 'distance-limited' ('magnitude-limited') sample is 15 (13).

The catalogs from which the samples were taken are incomplete - there is a relative deficiency of both higher recession velocity and fainter galaxies, as revealed through $V / V_{m}$ tests (see Paper II). These incompletenesses are especially severe for LINERs. In addition, many galaxies with low luminosity active nuclei are omitted from these catalogs (e.g. Ho, Filippenko \& Sargent 1995). Lastly, there is a relative deficiency of highly inclined galaxies, a well known result of obscuration of the active nucleus by the galaxy disk (e.g. Keel 1980; see fig. 5 of Paper II).

The statistical results of the distance- and magnitude-limited samples are very similar and we shall focus on the former sample in this paper. Six percent of the galaxies were detected. This detection statistic is similar to that in the recent survey of 33 AGN by Greenhill et al. (1997), which detected one new water vapor maser, and the Nobeyama survey of about 150 galaxies (Nakai, this meeting), which found three new masers.

When a sample of objects with a wide range of distances is detected over a limited range of flux density, plots involving luminosities tend to be artificially smeared along a line of unit slope. This potential problem of artificially induced correlations is particularly acute in the present sample, for which $94 \%$ of the galaxies are undetected in water emission. For this reason, we have limited our study to a comparison between the properties of maser-detected and maser-undetected galaxies.

\section{Results}

The parameters which are distributed differently between the maser-detected and -undetected galaxies are listed in Table 1, along with the probability that one parent population would generate the distributions of both detected and undetected galaxies. We include only those parameters for which this probability is $<0.05$. A range is given for each of these probabilities, based on the results of the K-S test and five other tests from the ASURV package, which includes treatment of upper limits (e.g. Feigelson \& Nelson 1985). Details are given in Paper II.

TABLE 1. Parameters distributed differently between detected and undetected galaxies in the distance-limited sample

\begin{tabular}{llllll}
\hline Parameter & Probability & Parameter & Probability & Parameter & Probability \\
\hline Recession Velocity & $<0.01-0.09$ & IRAS S $(12 \mu \mathrm{m})$ & $<0.01$ & IRAS S $(25 \mu \mathrm{m})$ & $<0.01$ \\
IRAS S $(60 \mu \mathrm{m})$ & $\leq 0.01$ & IRAS S $(60) / \mathrm{S}(100)$ & $0.01-0.03$ & $\mathrm{~S}_{n u c}(6 \mathrm{~cm})$ & $0.02-0.04$ \\
Galaxy Inclination & $0.03-0.09$ & Seyfert type & 0.01 & Hydrogen Column & See text \\
\hline
\end{tabular}

In evaluating the results of the statistical tests, it is helpful to bear in mind the following simplified situations. If all active galaxies had similar $\mathrm{H}_{2} \mathrm{O}$ maser luminosities, then we would tend to detect the nearer ones. If, on the other hand, the ratios of the maser luminosity to the luminosities in other wavebands were similar from galaxy to galaxy (i.e. the galaxies have similar spectral shapes, but widely differing intrinsic luminosities), then we would tend to detect the apparently brighter (in other wavebands) ones. In a dataset with a limited number of detections, it is difficult to distinguish 


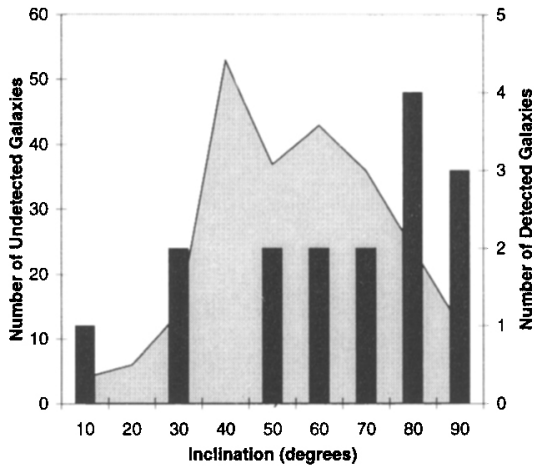

Figure 1. The distributions of galaxy disk inclination for the maser-detected and -undetected galaxies with recession velocities below $7,000 \mathrm{~km} \mathrm{~s}^{-1}$. The light shaded region represents the undetected galaxies (on the scale on the left. axis) in the distance-limited sample of Paper II plus 17 disk galaxies with recession velocity below $7,000 \mathrm{~km} \mathrm{~s}^{-1}$ undetected by Greenhill et al. (1997). The vertical bars represent the detected galaxies (on the scale on the right axis) from Paper II, Greenhill et al. (1997) and Hagiwara et al. (1997).

between these possibilities, since the nearer galaxies tend to be the apparently brighter. We first perform tests of these 'astrophysical expectations' before going on to more physically revealing correlations.

\subsection{RECESSION VELOCITY}

The maser-detected galaxies are nearer than the maser-undetected galaxies. The probabilities that one parent population would generate the distributions of both are 0.01 or less in three tests and 0.09 in a fourth test (see Paper II for details).

\subsection{FLUX DENSITIES}

The maser-detected galaxies have higher flux densities than the maser-undetected galaxies at $12 \mu \mathrm{m}$, $25 \mu \mathrm{m}, 60 \mu \mathrm{m}$ and $6 \mathrm{~cm}$. Although we have used IRAS fluxes, which have low spatial resolution, it is interesting that the probability that the detected galaxies are brighter than the undetected galaxies is only $\approx 90 \%$ or less at $100 \mu \mathrm{m}$. Since the shorter wavelength IRAS-detected emission is believed to originate in the nuclear region of Seyfert galaxies, while the longer wavelength emission probably originates in the galaxy disk, the observed correlations favor a relationship between water vapor maser power and nuclear mid-infrared power. Given these results, it is not surprising that the detected galaxies tend to be warmer in the $60 \mu \mathrm{m} / 100 \mu \mathrm{m}$ flux ratio than the non-detected.

The difference between the nuclear $6 \mathrm{~cm}$ fluxes of the maser-detected and -undetected galaxies has somewhat lower significance (Table 1). It is unlikely that this correlation reflects a direct relationship between 'seed' continuum photons and maser output since the nuclear centimeter wavelength emission of Seyfert galaxies is dominated by $100 \mathrm{pc}$-scale emission (e.g. Ulvestad \& Wilson 1989), much larger than the believed overall extent of the maser spots. The relationship could result if the sub-pc scale radio emission, responsible for the 'seed' photons, is correlated with the observed radio emission.

\subsection{GALAXY DISK INCLINATION}

Early in the survey, we noted (Braatz, Wilson \& Henkel 1996b) a trend for the galactic disks of maser-detected galaxies to be more highly inclined than those of maser-undetected galaxies with probability (as defined above) $\approx 0.05$. When the survey was completed, this probability had increased to $0.06-0.21$ and was thus much less significant. However, both of the galaxies (NGC 3735 and NGC 5793) detected by Greenhill et al. (1997) and Hagiwara et al. (1997) are within our distance limit and have high inclination. Including these detections, the probability that the maser-detected and -undetected galaxies have inclinations drawn from the same distribution drops to $0.03-0.09$ (Fig. 1). The only simple interpretation of this result is that a significant portion of the maser gain occurs in gas co-planar with the galaxy disk. 


\subsection{SEYFERT TYPE}

The most striking trend from our survey is that no Seyfert 1s were detected. Ten of the masers are found in Seyfert 2 galaxies and five are in LINERs. This difference in the detection rates is not a result of different distances for the two Seyfert types, both of which have mean distances of $53.0 \mathrm{Mpc}\left(\mathrm{H}_{0}=75 \mathrm{~km} \mathrm{~s}^{-1} \mathrm{Mpc}^{-1}\right)$. The LINERs are slightly closer - mean distance $37.3 \mathrm{Mpc}$. For most of the sample studied, Seyfert sub-types $(1.2,1.5$ etc.) are not available. However, very weak, broad $\mathrm{H} \alpha$ has been detected in some of the $\mathrm{H}_{2} \mathrm{O}$-maser detected galaxies, including NGC 1052, NGC 2639, NGC 4258 and possibly NGC 3079 (Ho et al. 1997). This result indicates that $\mathrm{H}_{2} \mathrm{O}$ maser emission can occur in galaxies with very weak or heavily obscured broad line regions. Of course, it is possible that all Seyfert 2 s contain such nuclei.

\subsection{EQUIVALENT HYDROGEN COLUMN}

Observations with ASCA (Awaki 1997; Turner et al. 1997) show that essentially all Seyfert 2s exhibit equivalent hydrogen columns (derived from photoelectric absorption of soft X-rays) to their nuclei above $10^{22.5} \mathrm{~cm}^{-2}$. The column densities to Seyfert $1 \mathrm{~s}$ are almost always below $10^{22} \mathrm{~cm}^{-2}$. The difference in detection rates between Seyfert $1 \mathrm{~s}$ and $2 \mathrm{~s}$ may thus be interpreted as an increasing probability of maser detection with increasing column to the nucleus. It is noteworthy that 3 out of the 6 galaxies listed by Awaki (1997) as having very high columns $-\mathrm{N}_{H} \geq 10^{24} \mathrm{~cm}^{-2}$-are detected as water vapor masers. However, these three galaxies - NGC 1068, NGC 4945 and Circinus - are all nearby, and this apparent increased probability of detection at very high column may instead be a result of their proximity.

\section{Conclusions}

The main conclusions of this work are:

i) LINERs are detected in water vapor maser emission at a similar rate to Seyfert 2 s, suggesting they are predominantly AGN rather than starbursts.

ii) The key parameter affecting water vapor maser production is the gas column to the nucleus. This is indicated by both the absence of detections among Seyfert $1 \mathrm{~s}$ and the trend for galaxies with higher equivalent hydrogen column (as measured by X-ray absorption) to be preferentially detected as masers. The trend for the detected galaxies to have higher inclination suggests that some maser amplification occurs in gas coplanar with the galaxy disk.

iii) The dependence of maser detectability on gas column cannot, on its own, distinguish between special geometries (e.g. disk) and large intrinsic differences of column density from galaxy to galaxy. iv) The tendency for maser-detected galaxies to have higher $12 \mu \mathrm{m}, 25 \mu \mathrm{m}$ and $60 \mu \mathrm{m}$ fluxes than non-detected ones suggests the maser and AGN luminosities are correlated.

\section{References}

Awaki, H. 1997, In 'Emission Lines in Active Galaxies: New Methods and Techniques," IAU Colloquium No. 159, Eds. B.M. Peterson, F-Z. Cheng \& A.S. Wilson, p44 (San Francisco: Astronomical Society of the Pacific)

Braatz, J. A., Wilson, A. S., \& Henkel, C., 1996a, ApJ Supplements, 106, 51 (Paper I)

Braatz, J. A., Wilson, A. S., \& Henkel, C., 1996b, In "Proceedings of the Oxford Torus Workshop" Ed. M. J. Ward, Vistas in Astronomy, 40, No. 1.

Braatz, J. A., Wilson, A. S., \& Henkel, C., 1997, ApJ Supplements, 110, 321 (Paper II)

Feigelson, E. D. \& Nelson, P. I. 1985, ApJ, 293, 192

Greenhill, L. J., Herrnstein, J. R., Moran, J. M., Menten, K. M. \& Velusamy, T. 1997, ApJ (Letts), in press

Hagiwara, Y., Kohno, K, Kawabe, R. \& Nakai, N. 1997, PASJ, 49, 171

Herrnstein, J. R., Moran, J. M., Greenhill, L. J., Diamond, P. J., Miyoshi, M., Nakai, N. \& Inoue, M. 1997, ApJ (Letts), 475, L17

Ho, L. C., Filippenko, A. V. \& Sargent, W. L. W. 1995, ApJ Supplements, 98, 477

Ho, L. C., Filippenko, A. V., Sargent, W. L. W. \& Peng, C. Y. 1997, ApJ Supplements (in press)

Huchra, J.P. 1993, private communication

Keel, W. 1980, AJ, 85, 198

Miyoshi, M., Moran, J. M., Herrnstein, J., Greenhill, L. J., Nakai, N., Diamond, P. \& Inoue, M. 1995, Nature, 373 , 127

Neufeld, D. A., Maloney, P. R. \& Conger, S. 1994, ApJ (Letts), 436, L127

Turner, T. J., George, I. M., Nandra, K. \& Mushotzky, R. F. 1997, Preprint

Ulvestad J. S. \& Wilson, A. S. 1989, ApJ, 343, 659

Véron-Cetty, M.P., \& Véron, P. 1991, E.S.O. Sci. Rep. 10 